Research report

\title{
What is important in being cured from depression? Discordance between physicians and patients (1)
}

\author{
Koen Demyttenaere ${ }^{\mathrm{a}, *}$, Anne-Françoise Donneau ${ }^{\mathrm{b}}$, Adelin Albert ${ }^{\mathrm{b}}$, Marc Ansseau ${ }^{\mathrm{c}}$, \\ Eric Constant ${ }^{\mathrm{d}}$, Kees van Heeringen ${ }^{\mathrm{e}}$ \\ a University Psychiatric Centre, University of Leuven, Campus Gasthuisberg, Leuven, Belgium \\ ${ }^{\mathrm{b}}$ Department of Medical Informatics and Biostatistics, University of Liège, CHU, Sart Tilman, Liège, Belgium \\ ${ }^{\mathrm{c}}$ Department of Psychiatry and Medical Psychology, University and CHU of Liège, CHU, Sart-Tilman, Liège, Belgium \\ ${ }^{\mathrm{d}}$ Department of Psychiatry, Catholic University of Louvain, Cliniques Universitaires Saint-Luc, Brussels, Belgium \\ e University Department of Psychiatry and Medical Psychology, Unit for Suicide Research, University of Ghent Hospital, Ghent, Belgium
}

\section{A R T I C L E I N F O}

\section{Article history:}

Received 15 June 2014

Received in revised form

1 December 2014

Accepted 2 December 2014

Available online 10 December 2014

\section{keywords:}

Depression

Antidepressants

Expectations

Outcome

\begin{abstract}
A B S T R A C T
Aims: The comparison of what physicians and patients consider important in being cured from depression.

Methods: 426 outpatients (in primary care and in psychiatric care) with a clinical diagnosis of major depression were included: at the start of antidepressant treatment, the importance of a range of items for being cured from depression (depressive, anxious and somatic symptoms, positive affect, functional impairment, quality of life) was assessed in physicians and patients separately and a ranking was made; after 3 months of treatment, the importance of these items for being cured from depression was re-assessed in the patients.

Results: The items ranked top 10 by physicians mainly contain depressive symptoms while those ranked top 10 by patients mainly contain positive affect items and this attention to positive affect even increases at 3 months follow-up and is higher in patients with recurrent depression than in patients with a first episode of depression. Somatic symptoms consistently get the lowest ranking, as well in physicians as in patients.

Conclusions: Physicians differ significantly from patients in what they consider important for 'being cured from depression': physicians mainly focus on alleviation of depressive symptoms while patients mainly focus on the restoration of positive affect.
\end{abstract}

(c) 2014 Published by Elsevier B.V.

\section{Introduction}

Changes in symptom severity during antidepressant treatment are usually assessed with observer rating scales: the 17-item Hamilton Depression Rating Scale (HDRS) or the 10-item Montgomery-Asberg Depression Rating Scale (MADRS), hence giving priority to the physician's view on change (Hamilton, 1960; Montgomery and Asberg, 1979). The purpose of the HDRS was developing a scale for assessing severity and change during treatment within a population of patients already diagnosed with depression. It is interesting that the purpose of the MADRS was sensitivity and accuracy of change during antidepressant treatment: the authors chose the 10 items (out of the 65 items of the CPRS - Comprehensive Psychiatric Rating Scale) that changed most during treatment with a variety of antidepressants with different mechanisms of action (mianserin,

\footnotetext{
* Corresponding author.

E-mail address: koen.demyttenaere@med.kuleuven.be (K. Demyttenaere).
}

amitriptyline, maprotiline and clomipramine) (Montgomery and Asberg, 1979; Asberg et al., 1978). For the assessment of the efficacy of antidepressants, regulatory bodies consistently request one of these 2 standard observer-rating depression scales to be used and physicians are trained to use them in clinical practice. It is important to mention that both scales were published even before DSM-III was introduced and the content of these scales hence does not fully represent the currently used diagnostic criteria for depression (American Psychiatric Association, 1980).

It is remarkable that the 2 core symptoms of the DSM diagnostic criteria are to a different degree included in the rating scales, but both symptoms do lose the 'core symptom' position they have in the diagnostic criteria. While sad or depressed mood is well represented in both scales, it is remarkable and/or problematic that anhedonia has a very marginal position in both scales.

The 17-item HDRS indeed pays more attention to negative affect items than to anhedonia: depressed mood (sadness, hopeless, helpless, worthlessness...hence not only referring to affect but also to cognitions), psychic anxiety (psychological: subjective 
tension and irritability, worrying) and somatic anxiety (Hamilton, 1960). Only one item is referring more or less to anhedonia: work and activities (thoughts and feelings of incapacity, fatigue or weakness, loss of interest in activities, hobbies or work, decrease in actual time spend in activities or decrease in productivity, stopping working... hence not only referring to anhedonia but also to functioning).

The same is true for the 10-item MADRS containing 3 negative affect items: apparent sadness (representing despondency, gloom and despair, (more than just ordinary transient low spirits), reported sadness (representing depressed mood, low spirits, despondency or feeling of being beyond help without hope) and inner tension (representing feelings of ill-defined discomfort, edginess, inner turmoil mounting to either panic, dread or anguish) (Montgomery and Asberg, 1979). And only one item is referring to anhedonia although in the higher scores it is referring to complete inability to feel positive as well as negative emotion: inability to feel (representing the subjective experience of reduced interest in the surrounding, or activities that normally give pleasure up to the experience of being emotionally paralyzed, inable to feel anger, grief or pleasure).

Two remaining questions are whether observer-rating indeed are superior to self-rating scales and whether the content of these observer rating scales really reflect the concerns of patients about outcome in depression treatment. It is known that discrepancies can exist between observer- and self-rating scales: in comparison to observer rating very severely depressed patients have been shown to underrate the severity of their depression while mildly depressed patients have been shown to overrate the severity of their depression (Möller, 2000). Discrepancies also exist between the content of the most frequently used rating scales and what patients expect from treatment: Zimmerman showed that, from a patient perspective, the rank order of the most important expectations from antidepressant treatment are first presence of positive mental health (optimism, vigor, self-confidence), second feeling like your usual, normal self, third return to usual level of functioning at work, home or school, fourth feeling in emotional control, fifth participating in and enjoying relationships with family and friends, and only sixth absence of symptoms of depression (negative affect) (Zimmerman et al., 2013). They developed the Remission from Depression Questionnaire (RDC), a 41-item self-report measure that assesses an array of features reported by patients as relevant to determining remission from depression, including positive mood (Zimmerman et al., 2013). Significant differences were found for HDRS remitted patients (observer rated) and RDQ remitted patients (self-rated) and depressed patients were shown to have a perspective of remission going beyond symptoms resolution and also including positive mental health or life satisfaction. On the same lines, another group developed the REMIT (Remission Evaluation and Mood Inventory Tool) questionnaire taking into account patient expectations: in predicting patient assessed recovery from depression, adding the 5 items of the scale (emotional control, contentedness, future seeming dark, ability to bounce back and happiness) to the Patient Health Questionnaire (PHQ, reflecting DSM criteria of depression) yielded an $11 \%$ increase in $R^{2}$ beyond the $60 \%$ yielded by the PHQ (Nease et al., 2011). This picture seems to be far different from what regulatory bodies or physicians expect from outcome and from what the HDRS or MADRS assess and suggests that a broader perspective should be considered when assessing outcome. Moreover, real life patients often have comorbid anxious and somatic symptoms, have excessive negative affect and lack of positive affect, present with functional impairment in their occupational, social and family life and report a low quality of life (Demyttenaere et al., 2010; Demyttenaere et al., 2009; Watson et al., 1988a, 1988b; De Fruyt and Demyttenaere, 2009). The two standard scales show some differences here: the HDRS covers a somewhat broader spectrum of symptoms including anxiety and neurovegetative (somatic) symptoms while the MADRS is more focused on the depressive symptomatology but neither scale covers the whole range of issues/symptoms mentioned above.

Therefore, a more comprehensive assessment of change is probably warranted and the present study investigates the concordance or divergence between what physicians and patients consider to be the most important issues in considering cure from depression.

\section{Methods}

\subsection{Ethics statement}

This research was performed according to the principlesù of the Declaration of Helsinki, and written informed consent was obtained from the participants after explanation of the nature of the study. The study was approved by the ethics committee of Hôpital Universitaire Saint-Luc in Woluwe (Brussels).

\subsection{Patient recruitment}

This is a prospective, non-interventional study conducted in Belgium between February 2010 and July 2011 in both general and specialized (psychiatric) practices. According to the study protocol, 150 physicians, specifically 75 general practitioners (GP) and 75 specialists (SP), were requested to enroll 4 clinically depressed patients. Any selected physician who declined to participate was replaced by another physician who previously participated in the DEsCRIBE $^{\mathrm{TM}}$ survey (Demyttenaere et al., 2011). At the end of the enrollment period, 453 patients had been included. Patients, male or female and older or equal to 18 years, suffering from a novel episode of clinical depression where the physician decided that treatment with an antidepressant was indicated and initiated were eligible for study participation. Patients unable to fill in questionnaires and/or treated for the current episode with an antidepressant for longer than 2 weeks were excluded $(N=27)$. Therefore, analyses were performed on 426 patients: 242 patients enrolled by 61 GPs, 211 patients enrolled by 57 psychiatrists. The mean number of patients included per physician was $4 \pm 2$ (range 1-7); $39 \%$ of GPs and $47 \%$ of psychiatrists enrolled less than 4 patients. The study protocol did not interfere with the treatment decisions of the physician.

\subsection{Data collection}

Data were collected from both patients and their physicians. At baseline patient's demographics, medical and psychiatric history, details on the choice of antidepressant and non-pharmaceutical treatment were collected and the physician assessed the clinical status for a given patient with the CGI-S (Clinical Global Impression of Severity) (Guy, 1976).

What physicians and patients found to be most important in being cured from depression was assessed with the DEsCRIBE ${ }^{\mathrm{TM}}$ questionnaire. This questionnaire contains 51 items measuring severity of depressive, anxious and somatic symptoms, positive affect, functional impairment and quality of life; the items had been taken from six validated scales: the Patient Health Questionnaire-Depression subscale (PHQ-depression); the Hospital Anxiety and Depression Scale-anxiety subscale (HADS-anxiety); the Patient Health Questionnaire-Somatic Symptoms severity subscale (PHQ-somatic); the Positive And Negative Affect Schedule-Positive Affect subscale (PANAS-positive); the Sheehan Disability Scale (SDS); and the Abbreviated World Health Organization QoL scale - psychological and social subscales (WHOQOL-BREF) (Kroenke et al., 2001; Zigmond and Snaith, 1983; Kroenke et al., 2002; 
Watson et al., 1988; Sheehan et al., 1996; WHO-QOL group, 1998). Each item is rated on a 5 -point Likert scale (where $1=$ not important for being cured from depression and $5=$ very important for being cured from depression).

The clinical status of the patient was assessed with the above mentioned scales: the patient indeed filled out the PHQ-depression, HADS-anxiety, PHQ-somatic, the PANAS-positive mood subscale, the SDS, and the WHOQOL-BREF at baseline and at 6 months of treatment (Kroenke et al., 2001; Zigmond and Snaith, 1983; Kroenke et al., 2002; Watson et al., 1988; Sheehan et al., 1996; WHO-QOL group, 1998). At 3 months the patient again completed the DEsCRIBE ${ }^{\mathrm{TM}}$ questionnaire (in order to test whether the change in clinical status changed his/her expectations from treatment). The scales were to be filled in by the patient in a separate room at the physician's practice and put in a sealed envelope before handing it over to the physician in order to avoid any influencing between physician and patient. Except for baseline assessments, the patient had to fill in the questionnaire before seeing the physician.

\subsection{Statistical analysis}

Results were summarized as mean and standard deviation (SD) for quantitative variables and scores; counts and proportions (\%) were used for categorical variables. Mean values were compared by oneway analysis of variance followed by multiple comparisons in case of several groups. Proportions were compared by the chi-squared test for contingency tables. The agreement between the physicians and the patients on the 51 DEsCRIBE $^{\mathrm{TM}}$ questionnaire items was studied. First, the means of the 51 DEsCRIBE ${ }^{\mathrm{TM}}$ items were sorted by decreasing order for the physicians and patients separately. Then, the agreement on each of the 51 DEsCRIBE ${ }^{\mathrm{TM}}$ items was assessed taking into account the matching between the patients and the physicians. Specifically, ranks were attributed to each of the $51 \mathrm{DEsCRIBE}^{\mathrm{TM}}$ items individually for each patient and for each physician. Based on these ranks, a pair patient-physician was defined to be in agreement for a DEsCRIBE $^{\mathrm{TM}}$ item if they both classified this item in first position (i.e., ranked in first position). The procedure was repeated for each DEsCRIBE item and for each patient-physician pair. The percentages of agreement in first position of importance in being cured from depression of the 51 DEsCRIBE ${ }^{\mathrm{TM}}$ items were sorted by decreasing order. The entire procedure was repeated for the ranking in last position of importance in being cured from depression. The above methodology was repeated according to some physician's and patient's characteristics.

Results were considered significant at the 5\% critical level $(p<$ $0.05)$. Calculations were always done on the maximum number of data available. All statistical calculations were performed using the SAS (version 9.2 for Windows) and S-PLUS (version 8.1) statistical packages.

\section{Results}

\subsection{Diagnostic and clinical characteristics of the study population}

Physicians had been asked to include patients with a diagnosis of clinical depression 'where treatment with an antidepressant was indicated and initiated'. Based on the PHQ-depression scale scores, $69.8 \%$ fulfilled criteria for major depression. The mean scores on the different scales are given in Table 1.

Based on the HADS-anxiety scale $71.1 \%$ were probable cases and $20.0 \%$ were doubtful cases for an anxiety disorder. Overall, $16.0 \%$ of the patients did not fulfill screening/diagnostic criteria for either a major depressive episode or an anxiety disorder, $12.4 \%$ fulfilled diagnostic criteria for a major depressive disorder, $14.5 \%$ fulfilled screening criteria for an anxiety disorder, and 57.1\% fulfilled screening/diagnostic criteria for both a major depressive episode and an
Table 1

Demographic characteristics and baseline psychometric scores ( $n=426$ patients).

\begin{tabular}{ll}
\hline Age (years) & $46.4 \pm 13.8$ \\
\hline Female gender & $65.7 \%$ \\
Number of previous episodes & $1.29 \pm 1.83$ \\
PHQ-depression score & $16.5 \pm 5.75$ \\
HADS-anxiety score & $12.9 \pm 3.94$ \\
PHQ-somatic score & $9.69 \pm 4.82$ \\
PANAS-positive score & $21.1 \pm 7.07$ \\
SDS global score & $19.2 \pm 6.81$ \\
WHOQOL-BREFpsychological & $14.5 \pm 3.88$ \\
WHOQOL-BREFsocial relationships & $8.36 \pm 2.54$ \\
\hline
\end{tabular}

anxiety disorder. When defining a 'clinically significant functional impairment' as a score of at least 4 on the 3 SDS items, $70.7 \%$ of the included patients fulfilled this criterion.

The CGI-severity shows that the percentage of moderately, markedly and severely ill was $23.7 \%, 49.0 \%$ and $18.1 \%$ respectively. The relation between CGI-severity and the other scales are illustrated in Fig. 1. The CGI-severity score was associated with a significantly different score on the PHQ-depression score $(p<0.0001)$, the HADSanxiety score $(p=0.0007)$, the global SDS score $(p<0.0001)$, the WHOQOL-BREF psychological $(p<0.0001)$ and social subscores $(p=0.017)$ but not with the PHQ-somatic score $(p=0.74)$ and not with the PANAS-positive score $(p=0.13)$.

Antidepressants were prescribed to all patients, and 35\% of included patients were already receiving psychotherapeutic treatment (36.1\% interpersonal psychotherapy, 34.2\% cognitive-behavior psychotherapy, 7\% psychoanalytic psychotherapy, 7\% family therapy and $16.5 \%$ other forms of psychotherapy).

\subsection{Importance of items for physicians and for patients in defining cure from depression}

\subsubsection{Ranking of items being important in defining cure from depression, as rated by physicians or rated by patients}

When comparing the rank order based on the importance of the different scales for considering the patient cured from depression, differences were observed between physicians and patients (Table 2). First, patients overall gave higher scores to each of the scales. Second, patients ranked quality of life and positive affect higher while physicians ranked functioning and depressive symptomatology higher. Somatic symptoms were given the lowest ranking as well by physicians as by patients.

These differences became even more obvious when looking at the rank order of the individual items (Table $3 a$ and $3 b$ ). Indeed, a more detailed look at the content of the single items, the physician rated top 10 items comprised 4 items reflecting positive affect (how satisfied you are with yourself, how much do you enjoy life, to what extent life is meaningful, how satisfied you are with personal relationships - all 4 from the WHOQOL-BREF) while patient rated top 10 items comprised 7 items reflecting positive affect (to what extent life is meaningful, how much do you enjoy life, how satisfied are you with yourself, how able are you to concentrate, how strong you feel, how satisfied you are with personal relationships, how active you feel 5 from the WHOQOL-BREF and 2 from the PANAS-positive). The top 5 items in the ranking by physicians contain 4 depressive symptoms and 1 impairment item while the top 5 items in the ranking by patients contain 4 positive affect items and 1 depressive symptom. The DEsCRIBE ${ }^{\mathrm{TM}}$ questionnaire was re-administered to the patients at 3 months follow-up (when the clinical status had improved): the top 10 items now comprised 9 positive affect items and only 1 depressive symptom (in the 10th position) (Table 4).

On the contrary, a high concordance between physicians and patients was observed on which items are the least important in 

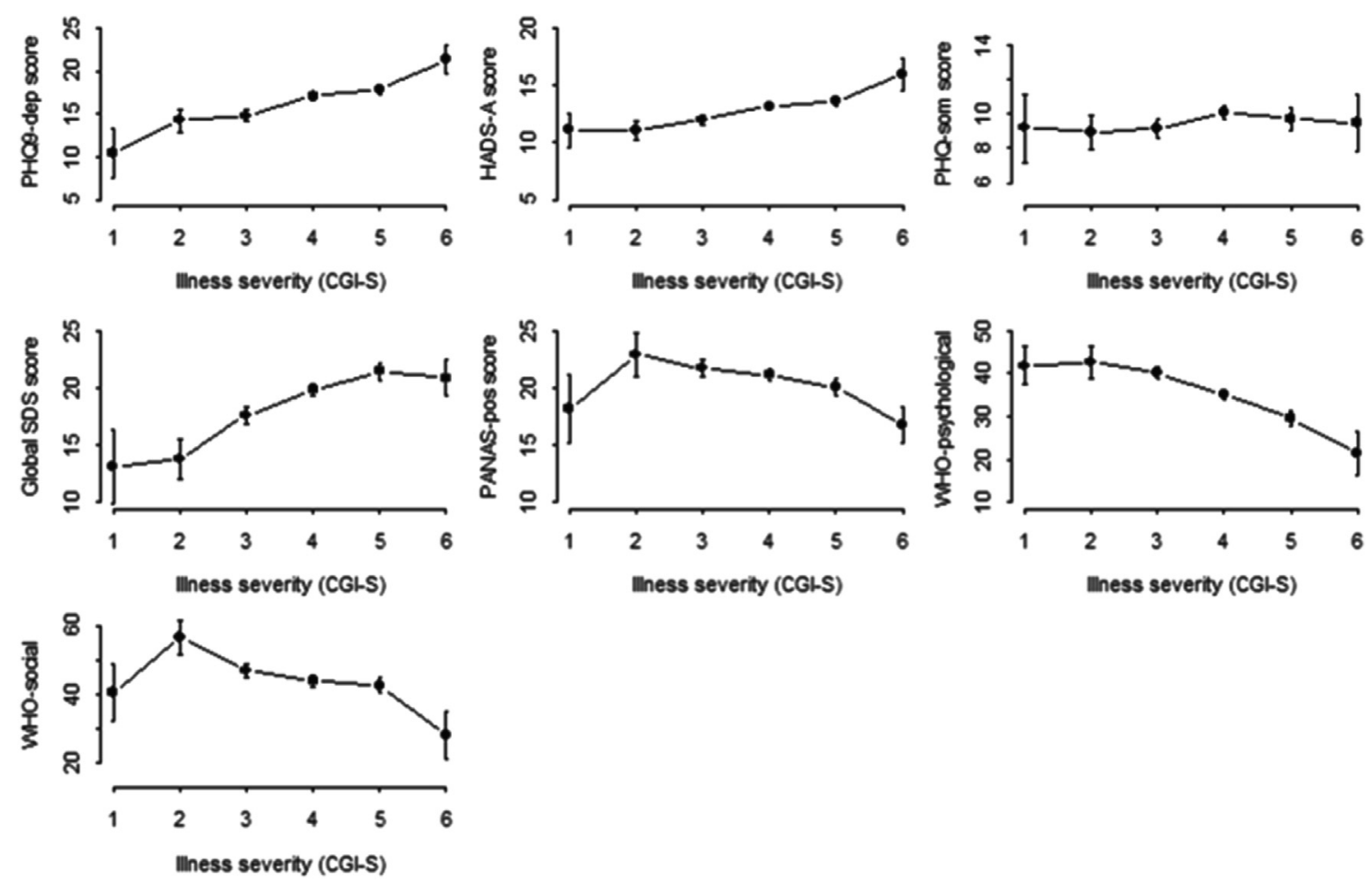

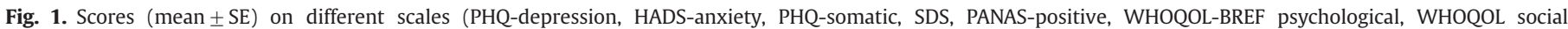
relationships) according to the CGI-severity.

Table 2

Importance of different scales for assessing cure (physicians versus patients).

\begin{tabular}{|c|c|c|c|}
\hline \multicolumn{4}{|c|}{$\begin{array}{l}\text { Mean ( } \pm \text { SD) scores of the six subscales reconstructed from the } 51 \text { DEsCRIBE }{ }^{\mathrm{TM}} \\
\text { items scored by the physician and the patient - by decreasing order. }\end{array}$} \\
\hline \multicolumn{4}{|c|}{ Physician $(n=453)$} \\
\hline Rank & Subscale & $N$ & Mean \pm SD \\
\hline 1 & SDS & 424 & $3.78 \pm 0.99$ \\
\hline 2 & WHOQOL-BREF & 413 & $3.59 \pm 0.77$ \\
\hline 3 & PHQ-depression & 407 & $3.52 \pm 0.88$ \\
\hline 4 & PANAS-positive & 410 & $3.35 \pm 0.89$ \\
\hline 5 & HADS-anxiety & 409 & $3.18 \pm 0.96$ \\
\hline 6 & PHQ-somatic & 389 & $2.24 \pm 0.79$ \\
\hline \multicolumn{4}{|c|}{ Patient $(n=453)$} \\
\hline 1 & WHOQOL-BREF & 413 & $4.00 \pm 0.74$ \\
\hline 2 & SDS & 424 & $3.89 \pm 1.09$ \\
\hline 3 & PANAS-positive & 410 & $3.86 \pm 0.85$ \\
\hline 4 & PHQ-depression & 407 & $3.66 \pm 1.00$ \\
\hline 5 & HADS-anxiety & 409 & $3.54 \pm 1.03$ \\
\hline 6 & PHQ-somatic & 389 & $2.70 \pm 1.02$ \\
\hline
\end{tabular}

the definition of being cured from depression: the 10 items with the lowest ranking were all somatic symptoms, as well in the patient ranking as in the physician ranking.

\subsubsection{Variables influencing the ranking of items being most} important in defining cure from depression

3.2.2.1. Clinical variables. Patients with a first depressive episode had 5 positive affect items in their top 10 most important items for defining cure from depression while patients with a recurrent episode had 8 positive affect items in their top 10 (the 5 found in patients with a first depressive episode - to what extent is life meaningful, how much do you enjoy life, how satisfied are you with yourself, how active are you, how able are you to concentrate
Table 3a

Rank order: 10 most important DEsCRIBE ${ }^{\mathrm{TM}}$ items scored by the physicians (baseline).

\begin{tabular}{|c|c|c|c|c|}
\hline Rank & Scale item & $N$ & Mean \pm SD & Item description \\
\hline 1 & WHOQOL-BREF & 422 & $4.10 \pm 1.13$ & $\begin{array}{l}\text { Negative feelings: blue mood, } \\
\text { despair, anxiety, depression }\end{array}$ \\
\hline 2 & PHQ-depressive & 418 & $4.09 \pm 1.16$ & $\begin{array}{l}\text { Feeling down, depressed or } \\
\text { hopeless }\end{array}$ \\
\hline 3 & PHQ9-depressive & 421 & $4.00 \pm 1.18$ & $\begin{array}{l}\text { Little interest or pleasure in } \\
\text { doing things }\end{array}$ \\
\hline 4 & SDS & 421 & $3.99 \pm 1.13$ & $\begin{array}{l}\text { Symptoms disrupted social life/ } \\
\text { leisure activities }\end{array}$ \\
\hline 5 & PHQ-depressive & 421 & $3.99 \pm 1.15$ & $\begin{array}{l}\text { Feeling tired or having little } \\
\text { energy }\end{array}$ \\
\hline 6 & WHOQOL-BREF & 423 & $3.92 \pm 1.13$ & $\begin{array}{l}\text { How satisfied are you with } \\
\text { yourself }\end{array}$ \\
\hline 7 & WHOQOL-BREF & 421 & $3.87 \pm 1.12$ & How much are you enjoying life \\
\hline 8 & SDS & 401 & $3.78 \pm 1.30$ & $\begin{array}{l}\text { Symptoms have disrupted your } \\
\text { work }\end{array}$ \\
\hline 9 & WHOQOL-BREF & 426 & $3.73 \pm 1.20$ & To what extent life is meaningful \\
\hline 10 & WHOQOL-BREF & 424 & $3.73 \pm 1.13$ & $\begin{array}{l}\text { How satisfied are you with your } \\
\text { personal relationships }\end{array}$ \\
\hline
\end{tabular}

- and 3 additional ones - how satisfied are you with relationships, how interested are you, how strong do you feel).

The content of the patient rated top 10 items was not influenced by the diagnostic status (reaching PHQ-depression criteria for a major depressive episode or not; reaching HADSanxiety screening criteria for an anxiety disorder or not), and was not influenced by patient gender. In order to further investigate whether baseline symptom profile influenced the patient in considering which factors are most important in defining cure from depression, a midsplit was performed for each scale and the ranking was compared in the subgroup of patients with a higher versus the subgroup of patients with lower than median score. Overall, the ranking appeared to be mainly independent from baseline symptom profile: the top 3 were for all subgroups quality of life, functioning and positive affect and the bottom 3 were 
Table 3b

Rank order: 10 most important DEsCRIBE ${ }^{\mathrm{TM}}$ items scored by the patients (baseline).

\begin{tabular}{|c|c|c|c|c|}
\hline Rank & Scale item & $N$ & Mean \pm SD & Item description \\
\hline 1 & WHOQOL-BREF & 426 & $4.35 \pm 0.98$ & To what extent life is meaningful \\
\hline 2 & WHOQOL-BREF & 421 & $4.35 \pm 0.99$ & How much do you enjoy life \\
\hline 3 & WHOQOL-BREF & 423 & $4.22 \pm 1.11$ & $\begin{array}{l}\text { How satisfied are you with } \\
\text { yourself }\end{array}$ \\
\hline 4 & WHOQOL-BREF & 422 & $4.13 \pm 1.05$ & How able are you to concentrate \\
\hline 5 & WHOQOL-BREF & 422 & $4.08 \pm 1.31$ & $\begin{array}{l}\text { Negative feelings: blue mood, } \\
\text { despair, anxiety }\end{array}$ \\
\hline 6 & PHQ-depression & 421 & $4.07 \pm 1.25$ & $\begin{array}{l}\text { Feeling tired or having little } \\
\text { energy }\end{array}$ \\
\hline 7 & PHQ-depression & 418 & $4.07 \pm 1.31$ & $\begin{array}{l}\text { Feeling down, depressed or } \\
\text { hopeless }\end{array}$ \\
\hline 8 & PANAS-positive & 423 & $4.05 \pm 1.09$ & Feeling strong \\
\hline 9 & WHOQOL-BREF & 424 & $4.04 \pm 1.10$ & $\begin{array}{l}\text { How satisfied are you with your } \\
\text { personal relationships }\end{array}$ \\
\hline 10 & PANAS-positive & 426 & $4.04 \pm 1.09$ & Feeling active \\
\hline
\end{tabular}

Table 4

Rank order: 10 most important DEsCRIBE ${ }^{\mathrm{TM}}$ items scored by the patients (at 3 months follow-up).

\begin{tabular}{|c|c|c|c|c|}
\hline Rank & Scale item & $N$ & Mean \pm SD & Item description \\
\hline 1 & WHOQOL-BREF & 324 & $4.21 \pm 0.98$ & How much do you enjoy life \\
\hline 2 & WHOQOL-BREF & 322 & $4.14 \pm 1.05$ & $\begin{array}{l}\text { How satisfied are you with } \\
\text { yourself }\end{array}$ \\
\hline 3 & WHOQOL-BREF & 328 & $4.13 \pm 1.02$ & $\begin{array}{l}\text { To what extent life is } \\
\text { meaningful }\end{array}$ \\
\hline 4 & WHOQOL-BREF & 327 & $4.03 \pm 1.04$ & How able are you to concentrate \\
\hline 5 & WHOQOL-BREF & 323 & $3.99 \pm 1.05$ & $\begin{array}{l}\text { How satisfied are you with your } \\
\text { personal relationships }\end{array}$ \\
\hline 6 & PANAS-positive & 327 & $3.94 \pm 1.03$ & Feeling active \\
\hline 7 & PANAS-positive & 324 & $3.92 \pm 1.01$ & Feeling strong \\
\hline 8 & PANAS-positive & 325 & $3.90 \pm 1.05$ & Interested \\
\hline 9 & PANAS-positive & 322 & $3.90 \pm 1.06$ & Enthusiastic \\
\hline 10 & PHQ-depression & 316 & $3.89 \pm 1.25$ & $\begin{array}{l}\text { Feeling down, depressed or } \\
\text { hopeless }\end{array}$ \\
\hline
\end{tabular}

always depression, anxiety and somatic symptoms. The only minor differences were a few subgroups where one scale moved one rank up or one rank down: in patients with a baseline higher than median quality of life, or a higher than median positive affect, or a higher than median anxiety level at baseline or a lower than median somatic symptom severity, positive affect got the second highest ranking (instead of the third for the other half of the patient population or for the entire study group); in patients with a higher than median functional impairment at baseline, functioning got the highest ranking (instead of the other half of the patient population or for the entire patient population).

3.2.2.2. Physician variables. The content of the patient rated top 10 items was not influenced by the gender of the physician or by the professional profile of the physician (psychiatrist or primary care physician).

\subsection{Concordance between physicians and patients on which item is most important in defining cure}

\subsubsection{Concordance between physicians and patients: ranking of} items being rated as the most important or the least important in defining cure from depression by both physicians and patients

Looking at the chance of a particular item to be found the most important by both physicians and patients, 'feeling down-depressedhopeless', 'negative feelings, despair' and 'little interest-anhedonia' were the top 3 having a $34.0 \%, 33.4 \%$ and $28.5 \%$ chance of being ranked as most important by both physicians and patients. Again, the 10 items having the highest chance of being ranked as least important by both physicians and patients were all somatic symptoms: the 3 items having the highest chance of being ranked as least important by both physicians and patients were 'fainting spells', 'constipation or loose bowels or diarrhea' and 'dizziness' (46.6\%, 24.6\% and $22.4 \%$ respectively).

\subsubsection{Variables influencing concordance between physician and} patients on which item is most important in defining cure

3.3.2.1. Clinical variables. Overall numerically higher physicianpatient concordance percentages on which 3 items are the most important in defining cure were found in patients with a probable major depressive episode than in patients without a probable major depressive episode (based on the PHQ-depression score cutoff): the percentages of concordance for the top 3 ranked items were $36.7 \%, 34.7 \%$ and $31.3 \%$ for patients fulfilling with a probable major depressive episode, and $33.9 \%, 28.1 \%$ and $28.1 \%$ for patients without a probable major depressive episode.

Even so, overall numerically higher physician-patient concordance percentages on which 3 items are the most important in defining cure were found in patients with a probable anxiety than in patients without a probable anxiety disorder (based on the HADS-anxiety score cut-off): the percentages of concordance for the top 3 ranked items were $36.4 \%, 34.9 \%$ and $29.1 \%$ for patients with a probable anxiety disorder, and $30.8 \%, 29.1 \%$ and $27.1 \%$ for patients without a probable anxiety disorder).

The concordance percentages on which items are most important in defining cure between physicians and patients with a first episode than between physicians and patients with a recurrent episode of major depression were comparable (top 3 ranked items: $35.8 \%, 32.4 \%$ and $30.0 \%$ versus $38.2 \%, 35.1 \%$ and $29.4 \%$ ).

3.3.2.2. Physician variables. Overall numerically higher physicianpatient concordance percentages on which 3 items are the most important in defining cure were found between psychiatrists and patients than between primary care physicians and patients (top 3 ranked items: $40.2 \%, 40.2 \%$ and $33.5 \%$ versus $28.6 \%, 27.4 \%$ and $27.4 \%$ ).

Overall slightly higher physician-patient concordance percentages on which 3 items are the most important in defining cure were found between female physicians and patients than between male physicians and patients (top 3 ranked items: $37.9 \%, 35.4 \%$ and $33.3 \%$ versus $34.8 \%, 32.1 \%$ and $29.2 \%$ ).

\section{Discussion}

Physicians were asked to enroll patients with major depressive disorder: only $69.8 \%$ of the included patients reached the threshold for 'probable depression' on the HADS depression subscale while 71.1\% reached the threshold for 'probable anxiety disorder' on the HADS anxiety subscale. While $16 \%$ of the patients even did not reach the threshold neither for 'probable depression' nor for 'probable anxiety disorder', 57.1\% of the patients reached the threshold for both 'probable depression' and 'probable anxiety disorder'. These figures are very close to those in the larger $(N=3353)$ naturalistic FINDER study where the reported percentages were $66.4 \%, 74.1 \%$, $15.3 \%$ and $55.9 \%$ respectively and again illustrate that physicians do not seem to differentiate well between depression and anxiety and again underscore the highly prevalent comorbidity between anxiety and depression (Demyttenaere et al., 2009).

When assessing the clinical global impression of severity (CGI) physicians apparently mainly take into account depressive and anxious symptoms, functional impairment and the psychological aspects of quality of life, but not the lack of positive affect nor the severity of somatic symptoms. This is indeed remarkable since lack 
of positive affect is a core item in the DSM criteria for major depression. And as mentioned above, it is also remarkable that the most frequently used scales to assess change in patients with major depression pay so little attention to items reflecting 'the lack of interest or pleasure' (Hamilton, 1960; Montgomery and Asberg, 1979). Somatic symptoms, although highly prevalent in mood and in anxiety disorders, also do not seem to influence the CGI.

Important differences were found in what physicians and what patients consider to be important in relief or cure from depression. This is already obvious when looking at the ranking of the scales: physicians emphasize the importance of functioning, quality of life and depressive symptoms while patients rather emphasize the importance of quality of life, functioning and positive affect. This becomes even more obvious when looking at the ranking of individual items: in the physicians top 10, only 4 items refer to positive mood while in the patients top 10,7 items refer to positive mood. The finding that restoration of positive mood is of primary importance for depressed patients is very much in accordance with the study published by Zimmerman, where the presence of positive mental health (optimism, vigor, self-confidence) got the highest ranking before feeling like your usual self, return to your usual level of functioning, feeling in emotional control, participating and enjoying relationships with family and friends...and the alleviation of depressive symptoms was ranked only sixth (Zimmerman et al., 2006). Further research should investigate whether the patient's 'broader look' at outcome also depends on the causal beliefs patients have on depression: the public, even when taking antidepressants, continues to hold a multifactorial causal model of depression with a primary emphasis on psychosocial issues beyond biogenetic and beyond psychiatric symptoms (Read et al., 2014a, 2014b; Schomerus et al., 2013). This could well influence the way they define cure from depression.

The present study illustrates that somatic symptoms consistently get the lowest ranking, as well by physicians as by patients: although physical symptoms are very common in major depression and in generalized anxiety disorder, and despite the highly prevalent overlap between depressive, anxious and somatic symptoms (in an epidemiological study in a primary care setting, depression was found in $6.6 \%$ but only $1.7 \%$ of the patients suffered from pure depression while $1.1 \%$ had comorbid anxiety disorder, $1.6 \%$ significant somatic symptoms and $2.3 \%$ had as well comorbid anxiety disorder) physicians as well as patients seem to have a dualistic view and once they the diagnosis of depression is established, somatic symptoms (associated with depression) no longer seem to be viewed as important (Löwe et al., 2008).

Diagnostic status (probable case of depression or not, comorbidity or not), patient or physician gender, physician specialty (GP or psychiatrist) did not change the physician's or patient's view on what is important for being cured from depression. The only variable that influenced the ranking was whether patients suffered from a first or a recurrent episode of depression: patients with recurrent depression even more strongly emphasized positive affect items as being important to feel cured from depression. Moreover, when the questionnaire was re-administered to the patients at 3 months follow-up, positive affect even got a more important rating: the top 10 items now included 9 positive affect items. A possible explanation for this finding could be that clinical improvement mainly results in a decrease of negative affect (depressive symptomatology) but to a lower degree in an increase in positive affect what is than more pronouncedly felt as a lack. And it could well be that patients with recurrent depression experienced an ongoing lack of positive affect after their first depressive episode, due to incomplete recovery or due to antidepressant side effects. SSRIs are often first line antidepressants and it has indeed been shown that patients reaching remission with serotonergic agents like fluoxetine, when compared to healthy controls, report less positive (and less negative) affect (Opbroek et al., 2002). A qualitative study on the emotional side effects of SSRIs also described a reduction of positive emotions, both reduced frequency and reduced intensity, part of what has been called 'emotional detachment' as a side effect of serotonergic agents: experiencing a restricted range of emotions that usually are part of everyday life (Price et al., 2009). This was further illustrated by a large study on the adverse emotional effects in 1829 New Zealanders taking antidepressants where sexual difficulties, feeling emotionally numb, feeling not like your usual self and reduction in positive feelings was reported in over $40 \%$ of patients (Read et al., 2014a, 2014b). And the study also showed that these emotional effects were not linked to the level of depression prior to taking antidepressants. Therefore, a perceived missing of positive emotions could influence what patients believe is important in being cured from depression in a subsequent depressive episode.

When physicians and patients did agree on an item being the most important for defining cure, feeling down-depressed-hopeless', 'negative feelings, despair' and 'little interest-anhedonia were the 3 items most frequently mentioned. Moreover, higher agreement percentages was found in patients with a probable depression (compared with no probable depression) and in patients with a probable anxiety disorder (compared with no probable anxiety disorder) suggesting that in more severe patients, agreement on which item is the most important for defining cure increases. On the contrary, the agreement on which items are the least important was highest for the somatic symptoms. Numerically higher percentages of agreement were found between psychiatrists and patients than between primary care physicians and patients and between female physicians and patients than between male physicians and patients.

In conclusion, physicians and patients seem to differ in their ranking of what is now most important for cure in depression. Physicians pay more attention to depressive symptoms and in assessing global severity they mainly take into account depressive and anxious symptom severity, functional impairment and some aspects of quality of life but not positive affect and not somatic symptoms severity. Patients, on the contrary, pay more attention to positive affect. After 3 months of treatment, patients even pay higher attention to positive affect as do patients with recurrent depression (compared to patients with a first episode of depression). Agreement upon which symptoms are the most important in defining cure seems to be higher in cases of a major depressive episode or of an anxiety disorder than in cases with subthreshold symptomatology.

\section{Role of funding source}

Lundbeck Belgium provided the logistical support provided for performing this study; there was complete independence of researchers in the collection, analysis, and interpretation of the data and in the writing of the report, and in the decision to submit the paper for publication.

\section{Conflict of interest}

All authors have completed the Unified Competing Interest form.

\section{Acknowledgment}

All authors participated in conceiving and designing the study. Anne-Françoise Donneau and Adelin Albert analyzed the data and Koen Demyttenaere coordinated the writing of the manuscript. All authors have completed the Unified Competing Interest form and declare that (1) A-F. D. and A.A. had financial support from Lundbeck Belgium for the statistical analysis, (2) there are no relationships that might have an interest in the submitted work in the previous 3 years, (3) their spouses, partners, or children have no financial relationships that may be relevant for the submitted work; and (4) they have no non-financial interests that may be relevant to the submitted work. 


\section{References}

American Psychiatric Association, 1980. Diagnostic and Statistical Manual of Mental Disorders, Third ed. (DSM-III).

Asberg, M., Montgomery, S.A., Perris, C., Schalling, D., Sedvall, G., 1978. A comprehensive psychopathology rating scale. Acta Psychiatr. Scand. Suppl. 271, 5-27.

De Fruyt, J., Demyttenaere, K., 2009. Quality of Life measurment in antidepressant trials. is there an added value? Psychother. Psychosom. 78 (4), 212-219.

Demyttenaere, K., Verhaeghen, A., Dantchev, N., Grassi, L., Montejo, A.L., Perahia, D.G., Quail, D., Reed, C., Tylee, A., Bauer, M., 2009. Caseness for depression and anxiety in a depressed outpatient population: symptomatic outcome as a function of baseline diagnostic categories. Prim. Care Companion J. Clin. Psychiatry 11 (6), 307-315.

Demyttenaere, K., Reed, C., Quail, D., Bauer, M., Dantchev, N., Montejo, A.L., Monz, B., Perahia, D., Tylee, A., Grassi, L., 2010. Presence and predictors of pain in depression: results from the FINDER study. J. Affect. Disord. 125 (1-3), 53-60.

Demyttenaere, K., Ansseau, M., Constant, E., Albert, A., Van Gassen, G., van Heeringen, K., 2011. Do general practitioners and psychiatrists agree about defining cure from depression. The DEsCRIBE ${ }^{\mathrm{TM}}$ survey. BMC Psychiatry11160 http://dxdoi.org/10.1186/1471-244X-11-169.

Guy, W. (Ed.), 1976. ECDEU Assessment Manual for Psychopharmacology. US Department of Heath, Education, and Welfare Public Health Service Alcohol, Drug Abuse, and Mental Health Administration, Rockville, MD.

Hamilton, M., 1960. A rating scale for depression. J. Neurol. Neurosurg. Psychiatry 23, 56-62.

Kroenke, K., Spitzer, R.L., Williams, J.B., 2001. The PHQ-9: validity of a brief depression severity measure. J. Gen. Intern. Med. 16, 606-613.

Kroenke, K., Spitzer, R.L., Williams, J.B.W., 2002. The PHQ-15: validity of a new measure for evaluating the severity of somatic symptoms. Psychosom. Med. 64, 258-266.

Löwe, B., Spitzer, R.L., Williams, J.B., Mussell, M., Schellberg, D., Kroenke, K., 2008. Depression, anxiety and somatization in primary care: syndrome overlap and functional impairment. Gen. Hosp. Psychiatry 30 (3), 191-199.

Möller, H.J., 2000. Rating depressed patients: oberver- versus self-assessment. Eur. Psychiatry 15, 160-172.

Montgomery, S.A., Asberg, M., 1979. A new depression scale designed to be sensitive to change. Br. J. Psychiatry 134, 382-389.
Nease Jr, D.E., Aikens, J.E., Klinkman, M.S., Kroenke, K., Sen, A., 2011. The remission evaluation and mood inventory tool. Gen. Hosp. Psychiatry 33 (3), 279-286.

Opbroek, A., Delgado, P.L., Laukes, C., Mc Gahney, C., Katranis, J., Moreno, F.A. Manber, R., 2002. Emotional blunting associated with SSRI-induced sexual dysfuntion. Do SSRIs inhibit emotional responses? Int. J. Neuropsychopharmacol. 5 (2), 147-151.

Price, J., Cole, V., Goodwin, G.M., 2009. Emotional side-effects of selective serotonin reuptake inhibitors: qualitative study. Br. J. Psychiatry 195, 211-217.

Read, J., Cartwright, C., Gibson, K., 2014a. Adverse emotional and interpersona effects reported by 1829 New Zealanders while taking antidepressants. Psychiatry Res. (216), 67-73.

Read, J., Cartwright, C., Gibson, K., Shiels, C., Haslam, N., 2014b. Beliefs of people taking antidepressants about causes of depression and reasons for increased prescribing rates. J. Affect. Disord. 168, 236-242.

Schomerus, G., Matschinger, H., Angermeyer, M.C., 2013. Causal beliefs of the public and social acceptance of persons with mental illness. Psychol. Med. 44 304-314.

Sheehan, D.V., Harnett-Sheehan, K., Raj, B.A., 1996. The measurement of disability. Int. Clin. Psychopharmacol. 11 (Suppl 3), S89-S95.

Watson, D., Clark, L.A., Carey, G., 1988a. Positive and negative affectivity and their relation to anxiety and depressive disorder. J. Abnorm. Psychol. 97 (3), 346-353.

Watson, D., Clark, L.A., Tellegen, A., 1988b. Development and validation of brief measures of positive and negative affect: the PANAS scales. J. Personal. Soc Psychol. 54 (6), 1063-1070.

The WHOQOL Group, 1998. Development of the World Health Organization WHOQOL-BREF quality of life assessment. Psychol. Med. 28 (3), 551-558.

Zigmond, A.S., Snaith, R.P., 1983. The Hospital Anxiety and Depression Scale. Acta Psychiatr. Scand. 67, 361-370.

Zimmerman, M., Mc Glinshey, J.B., Posternak, M.A., Friedman, M., Attiullah, N., Boerescu, D., 2006. How should remission from depression be defined? The depressed patient's perspective. Am. J. Psychiatry 163 (1), 148-150.

Zimmerman, M., Martinez, J., Attiullah, N., Friedman, M., Toba, C., Boerescu, D. Ragheb, M., 2013. A new type of scale for determining remission from depression: the Remission from Depression Questionnaire. J. Psychiatr. Res. 47, 78-82. 